Article

\title{
Qualitative and Quantitative Analysis of Phenolic Acids, Flavonoids and Iridoid Glycosides in Yinhua Kanggan Tablet by UPLC-QqQ-MS/MS
}

\author{
Yu Lin *, Wen Xu, Mingqing Huang, Wei Xu, Huang Li, Miao Ye, Xun Zhang and Kedan Chu \\ College of Pharmacy, Fujian University of Traditional Chinese Medicine, Fuzhou 350122, China; \\ E-Mails: xwfjlab@163.com (W.X.); huangmingqing3413@gmail.com (M.H.); \\ xwfjtcm@sina.com (W.X.); lihuang3413@gmail.com (H.L.); ymfjtcm@163.com (M.Y.); \\ zxfjtcm@gmail.com (X.Z.); chukedan@gmail.com (K.C.) \\ * Author to whom correspondence should be addressed; E-Mail: lyfjtcm@163.com or \\ yulam@fjtcm.edu.cn; Tel./Fax: +86-591-2286-1661.
}

Academic Editor: Derek J. McPhee

Received: 6 April 2015 / Accepted: 19 June 2015 / Published: 3 July 2015

\begin{abstract}
A simple, rapid and specific ultra-performance liquid chromatography-triple quadrupole mass spectrometry method was developed for the analysis of 29 bioactive components (10 phenolic acids, 16 flavonoids, and three iridoid glycosides) in Yinhua Kanggan tablet (YHKGT), a herbal prescription used for treating upper respiratory infections, fevers, coughs and pharyngalgia. The separation was successfully achieved using a Waters Cortecs UPLC C18 column $(50 \times 2.1 \mathrm{~mm}, 1.6 \mu \mathrm{m})$ and gradient elution with water- $0.1 \%$ formic acid and acetonitrile. Polarity switching mode was used in the optimization of multiple reaction monitoring conditions. The analytical method was validated for linearity, precision and accuracy. Calibration curves for the 29 marker compounds showed good linear regression $(r>0.9982)$. The limits of detection (LOD) and limits of quantification (LOQ) for the 29 analytes were in the range of $0.03-4.99 \mathrm{ng} / \mathrm{mL}$ and $0.16-14.87 \mathrm{ng} / \mathrm{mL}$, respectively. The relative standard deviation (RSD) values of intra-day precision, inter-day precision, repeatability, and stability were less than $2.79 \%$, $4.87 \%, 4.18 \%$ and $4.71 \%$, respectively. The recoveries of the 29 marker compounds were in the range of $94.67 \%-104.78 \%$ (RSD $\leq 4.72 \%$ ). These results have shown that this developed method was efficient for the quality evaluation of YHKGT.
\end{abstract}


Keywords: Yinhua Kanggan tablet; ultra-performance liquid chromatography; triple quadrupole mass spectrometry; quality control

\section{Introduction}

Recently, herbal medicines have received great interest and have been used as an important part of health care in the anti-viral treatment field since they have relatively few side-effects compared to modern therapeutics [1]. Yinhua Kanggan tablet (YHKGT), a well-known traditional Chinese medicinal preparation, containing Lonicera japonica (Jinyinhua), Cyrtomium fortunei (Guanzhong), Melicope pteleifolia (Sanchaku), Vitex negundo var. cannabifolia (Mujinggen), Tadehagi triquetrum (Hulucha), and Mussaenda pubescens var. alba (Shangancao), has been widely used in China to treat upper respiratory infections, fevers, coughs and pharyngalgia [2]. Previous studies have indicated that three types of compounds, including phenolic acids, flavonoids and iridoids, are responsible for the overall therapeutic effects of Jinyinhua as well as Mujinggen [3-9]. However, relatively little research has been done in regard to the chemical constituents and pharmacological effects of the other herbal ingredients in YHKGT [10-12].

Although herbal medicines are increasingly being understood and accepted by more and more people around the world, the problem of quality control remains one of the major obstacles for their internationalization. A published paper has described a high performance liquid chromatographydiode-array detector (HPLC-DAD) method for the determination of the content of chlorogenic acid, one of the major components in YHKGT [13], but the efficacy of YHKGT should be associated with the synergistic or interactive action of various types of compounds derived from its component herbs rather than only one of them. Actually, the present quality control method severely restricts the clinical applications and in-depth study of YHKGT, therefore, it is of great significance to develop a more sensitive and efficient analytical method for the determination of more bioactive components in YHKGT for its quality assurance. Moreover, the aforementioned three types of active compounds of YHKGT should be selected for the quality control analysis.

In the present study, we have developed and validated for the first time a polarity switching ultra-performance liquid chromatography coupled with triple quadrupole mass spectrometry (UPLC-QqQ-MS) method for the rapid simultaneous determination of 29 active components (10 phenolic acids, 16 flavonoids and three iridoid glycosides) in YHKGT. Thirteen batches of YHKGT were collected for the analysis. Additionally, to ensure the accuracy and the sensitivity of quantification, 2-hydroxycinnamic acid, liquiritin, and albiflorin were employed as internal standards for phenolic acids, flavonoids, and iridoids, respectively.

\section{Results and Discussion}

\subsection{Optimization of Sample Preparation}

In order to achieve optimal extraction efficiency, the variables involved in the extraction, such as extract solvent and extract method, were optimized. Due to the different polarity and water-solubility 
of some analytes, $70 \%$ aqueous methanol was chosen as the extraction solvent. To find the best extraction method, ultrasonic extraction, refluxing and Soxhlet extraction were selected because of their relatively shorter extraction time than percolation and maceration. The results suggested that ultrasonic extraction was simpler and more efficient than refluxing and Soxhlet extraction in extracting typical compounds 1-29 using 70\% aqueous methanol as extraction solvent (Figure S1). Moreover, to obtain the optimal extraction efficiency, the effects of different factors including the different concentrations of methanol-water solution $(30 \%, 50 \%, 70 \%$ and $90 \%)$, solvent volume $(10,50,100$ and 150 times) and extraction time $(15,30,45$ and $60 \mathrm{~min})$ on the extraction performance were evaluated. An almost equal amount of sample $(0.2 \mathrm{~g})$ was extracted and analyzed using the described procedure. As a result, compared with the extraction yields of different factors of the extraction solution, volume and extraction time for 29 typical compounds, it was found that ultrasonic extraction with 100 times the volume of $70 \%$ methanol-water for $30 \mathrm{~min}$ for one time was an optimum method to prepare the sample solution (Figures S2-S4).

\subsection{Optimization of Chromatographic Conditions}

The chromatographic conditions were optimized to improve the resolution and sensitivity and shorten the analysis time. Different mobile phases including methanol-water and acetonitrile-water were examined. Acetonitrile-water was found to produce better peak shapes than methanol-water. Interestingly, using 5\% methanol to water could increase the resolution of several isomers such as schaftoside and isoschaftoside. Moreover, it was found that formic acid was not only beneficial to improving the chromatographic separation, but also in improving the ionization efficiency of analytes. In addition, due to their similar structures, retention time and ionization response in the negative ion mode, 2-hydroxycinnamic acid, liquiritin and albiflorin were chosen as internal standards for phenolic acids, flavonoids and iridoids, respectively.

In order to develop a sensitive and accurate quantitative method, the MS/MS fragmentation for each analyte was investigated by direct infusion of the single standard solution into the mass spectrometer $(-)$-ESI and (+)-ESI source to optimize MS parameters, including product ion, cone voltage, and collision energy, the product ion of each analytes. The negative ion mode was found to be more suitable for flavonoids and iridoid glycosides analyses, while positive ion mode was found to be more suitable for phenolic acids. Therefore, ion polarity switching mode was used in the optimization of MRM conditions in the quantitative analysis. The optimum results are shown in Table 1 and the MRM chromatograms of the 29 markers are shown in Figure 1A. LC/MS chromatograms of analytes from a real sample is presented in Figure 1B. 
Table 1. Retention time, related MS data of 29 investigated compounds and three internal standards in the UPLC-QqQ MS analysis.

\begin{tabular}{|c|c|c|c|c|c|c|}
\hline Compounds & $\begin{array}{c}\mathbf{t}_{\mathrm{R}} \\
(\mathrm{min})\end{array}$ & $\begin{array}{c}\text { Precursor } \\
\text { Ion }(m / z)\end{array}$ & $\begin{array}{l}\text { Product } \\
\text { Ion }(m / z)\end{array}$ & $\begin{array}{c}\text { Cone } \\
\text { Voltage (V) }\end{array}$ & $\begin{array}{c}\text { Collision } \\
\text { Energy (eV) }\end{array}$ & Polarity \\
\hline Protocatechuic acid & 2.52 & 155 & 93 & 25 & 12 & Positive \\
\hline Neochlorogenic acid & 3.07 & 355 & 163 & 20 & 18 & Positive \\
\hline Protocatechualdehyde & 3.85 & 139 & 93 & 25 & 15 & Positive \\
\hline$p$-Hydroxybenzoic acid & 4.25 & 139 & 121 & 15 & 10 & Positive \\
\hline Chlorogenic acid & 5.15 & 355 & 163 & 20 & 18 & Positive \\
\hline Cryptochlorogenin acid & 5.61 & 355 & 163 & 20 & 18 & Positive \\
\hline Caffeic acid & 5.63 & 181 & 163 & 20 & 12 & Positive \\
\hline Swertiamarin & 5.99 & 419 & 179 & 20 & 12 & Negative \\
\hline Sweroside & 6.82 & 403 & 179 & 25 & 12 & Negative \\
\hline Schaftoside & 7.6 & 563 & 443 & 30 & 28 & Negative \\
\hline Agnuside & 7.93 & 465 & 285 & 50 & 22 & Negative \\
\hline Isoschaftoside & 7.97 & 563 & 443 & 30 & 28 & Negative \\
\hline Flavosativaside & 8.00 & 593 & 413 & 50 & 22 & Negative \\
\hline Vitexin $2 "$-rhamnoside & 8.28 & 577 & 413 & 40 & 25 & Negative \\
\hline Rutin & 8.33 & 609 & 300 & 35 & 35 & Negative \\
\hline Vitexin & 8.35 & 431 & 311 & 45 & 20 & Negative \\
\hline Hyperoside & 8.42 & 463 & 300 & 45 & 28 & Negative \\
\hline Isoquercitrin & 8.53 & 463 & 300 & 45 & 28 & Negative \\
\hline Luteoloside & 8.58 & 447 & 285 & 50 & 28 & Negative \\
\hline Isochlorogenic acid B & 8.88 & 517 & 163 & 20 & 22 & Positive \\
\hline Kaempferol-3-O-rutinoside & 8.99 & 593 & 285 & 35 & 30 & Negative \\
\hline Isochlorogenic acid A & 9.04 & 517 & 163 & 20 & 22 & Positive \\
\hline Astragalin & 9.19 & 447 & 285 & 35 & 22 & Negative \\
\hline Apigenin-7-glucoside & 9.25 & 431 & 267 & 55 & 35 & Negative \\
\hline Isochlorogenic acid C & 9.26 & 517 & 163 & 20 & 22 & Positive \\
\hline Luteolin & 9.48 & 285 & 133 & 50 & 32 & Negative \\
\hline Quercetin & 9.53 & 301 & 151 & 40 & 25 & Negative \\
\hline Apigenin & 9.59 & 269 & 151 & 45 & 32 & Negative \\
\hline Casticin & 9.97 & 373 & 343 & 35 & 22 & Negative \\
\hline Albiflorin (IS1) & 6.85 & 525 & 121 & 25 & 25 & Negative \\
\hline Liquiritin (IS2) & 8.28 & 417 & 255 & 25 & 20 & Negative \\
\hline 2-Hydroxycinnamic acid (IS3) & 9.22 & 165 & 123 & 15 & 12 & Positive \\
\hline
\end{tabular}



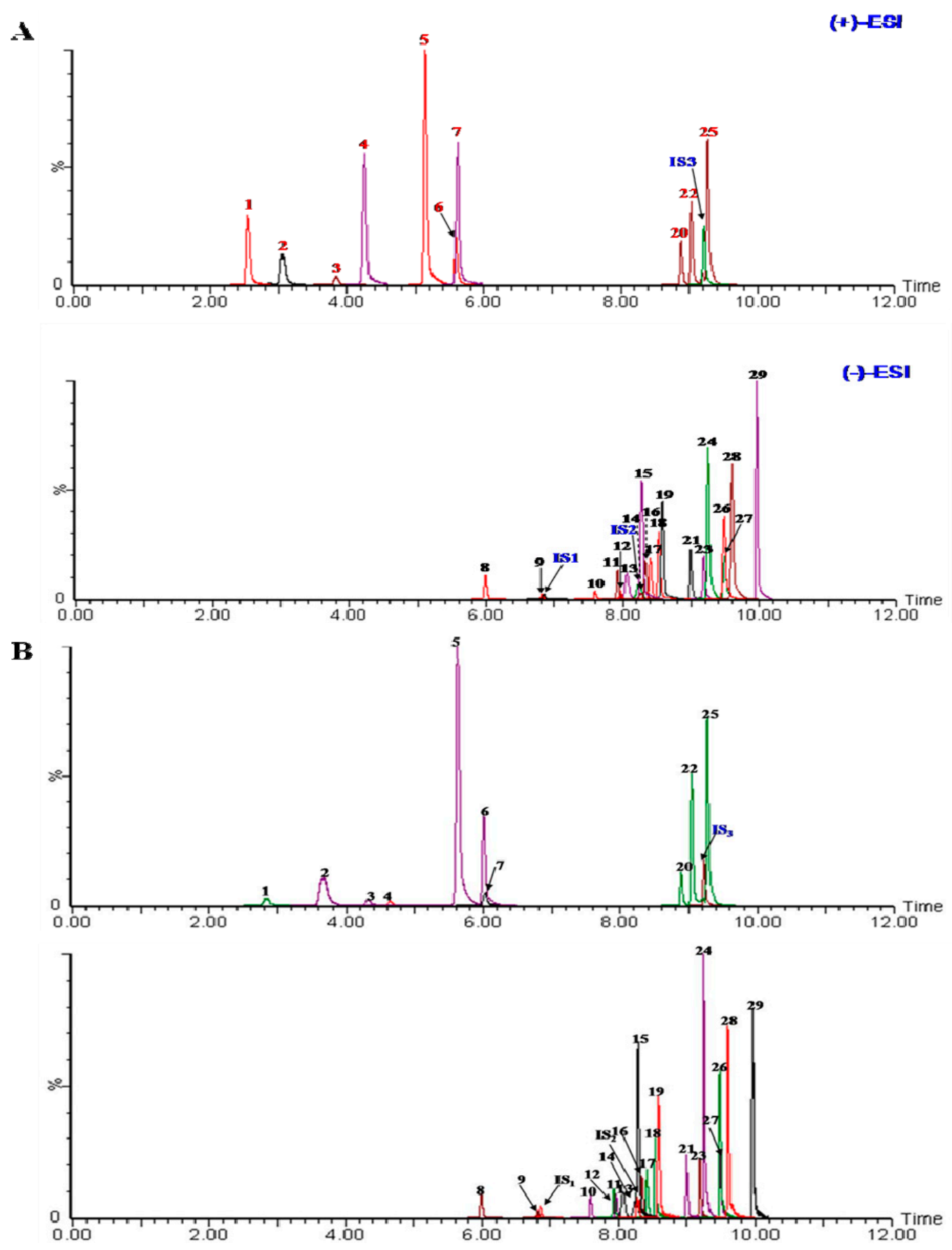

Figure 1. LC-MS/MS MRM chromatogram of 29 target standards(included 3 internal standards) (A) and samples (B): (1) protocatechuic acid; (2) neochlorogenic acid; (3) protocatechualdehyde; (4) 4-hydroxybenzoic acid; (5) chlorogenic acid; (6) cryptochlorogenin acid; (7) caffeic acid; (8) swertiamarin; (9) sweroside; (10) schaftoside; (11) agnuside; (12) isoschaftoside; (13) flavosativaside; (14) vitexin 2"-rhamnoside; (15) rutin; (16) vitexin; (17) hyperoside; (18) isoquercitrin; (19) luteoloside; (20) isochlorogenic acid B; (21) kaempferol-3-O-rutinoside; (22) isochlorogenic acid A; (23) astragalin; (24) apigenin-7glucoside; (25) isochlorogenic acid C; (26) luteolin; (27) quercetin; (28) apigenin; (29) casticin; (internal standard 1, IS 1 ) albiflorin; (IS 2 ) liquiritin; (IS $\mathbf{3})$ 2-hydroxycinnamic acid. 


\subsection{Identification of Compounds with UPLC-MS/MS}

The established analytical method was applied to identify the 29 compounds in YHKGT. The structures were unambiguously assigned based on their retention times and MS spectra of the reference standards (Table S1, Supplementary Information). The ESI mass spectra gave characteristic quasi-molecular ions of protocatechuic acid $\left([\mathrm{M}+\mathrm{H}]^{+}\right.$ion at $m / z$ 155), neochlorogenic acid $\left([\mathrm{M}+\mathrm{H}]^{+}\right.$ion at $\left.m / z 355\right)$, protocatechualdehyde $\left([\mathrm{M}+\mathrm{H}]^{+}\right.$ion at $m / z$ 139), $p$-hydroxybenzoic acid $\left([\mathrm{M}+\mathrm{H}]^{+}\right.$ion at $m / z$ 139), chlorogenic acid $([\mathrm{M}+\mathrm{H}]+$ ion at $m / z 355)$, cryptochlorogenin acid $\left([\mathrm{M}+\mathrm{H}]^{+}\right.$ion at $\mathrm{m} / z$ 355), caffeic acid $\left([\mathrm{M}+\mathrm{H}]^{+}\right.$ion at $\left.m / z 181\right)$, swertiamarin $\left([\mathrm{M}-\mathrm{H}+\mathrm{HCOOH}]^{-}\right.$ion at $\left.m / z 419\right)$, sweroside $\left([\mathrm{M}-\mathrm{H}+\mathrm{HCOOH}]^{-}\right.$ion at $\mathrm{m} / z$ 403), schaftoside $\left([\mathrm{M}-\mathrm{H}]^{-}\right.$ion at $\left.m / z 563\right)$, agnuside $\left([\mathrm{M}-\mathrm{H}]^{-}\right.$ion at $m / z 465)$, isoschaftoside $\left([\mathrm{M}-\mathrm{H}]^{-}\right.$ion at $\left.m / z 563\right)$, flavosativaside $\left([\mathrm{M}-\mathrm{H}]^{-}\right.$ion at $\mathrm{m} / z$ 593), vitexin $2^{\prime \prime}$-rhamnoside $\left([\mathrm{M}-\mathrm{H}]^{-}\right.$ion at $\left.m / z 577\right)$, rutin $\left([\mathrm{M}-\mathrm{H}]^{-}\right.$ion at $\left.m / z 609\right)$, vitexin $\left([\mathrm{M}-\mathrm{H}]^{-}\right.$ion at $\mathrm{m} / \mathrm{z} 431)$, hyperoside $\left([\mathrm{M}-\mathrm{H}]^{-}\right.$ion at $\left.\mathrm{m} / \mathrm{z} 463\right)$, isoquercitrin $\left([\mathrm{M}-\mathrm{H}]^{-}\right.$ion at $\left.\mathrm{m} / z 463\right)$, luteoloside $\left([\mathrm{M}-\mathrm{H}]^{-}\right.$ion at $\left.m / z 447\right)$, isochlorogenic acid $\mathrm{B}\left([\mathrm{M}+\mathrm{H}]^{+}\right.$ion at $\left.m / z 517\right)$, kaempferol-3-O-rutinoside $\left([\mathrm{M}-\mathrm{H}]^{-}\right.$ion at $\left.m / z 593\right)$, isochlorogenic acid $\mathrm{A}\left([\mathrm{M}+\mathrm{H}]^{+}\right.$ion at $\left.m / z 517\right)$, astragalin $\left([\mathrm{M}-\mathrm{H}]^{-}\right.$ion at $\mathrm{m} / \mathrm{z} 447)$, apigenin-7-glucoside $\left([\mathrm{M}-\mathrm{H}]^{-}\right.$ion at $\left.\mathrm{m} / \mathrm{z} 431\right)$, isochlorogenic acid $\mathrm{C}\left([\mathrm{M}+\mathrm{H}]^{+}\right.$ion at $\mathrm{m} / \mathrm{z}$ 517), luteolin $\left([\mathrm{M}-\mathrm{H}]^{-}\right.$ion at $\left.m / z 285\right)$, quercetin $\left([\mathrm{M}-\mathrm{H}]^{-}\right.$ion at $\left.\mathrm{m} / \mathrm{z} 301\right)$, apigenin $\left([\mathrm{M}-\mathrm{H}]^{-}\right.$ion at $m / z 267)$, and casticin $\left([\mathrm{M}-\mathrm{H}]^{-}\right.$ion at $\left.m / z 373\right)$.

Moreover, MS/MS analysis gave characteristic fragmentation behavior of the 29 compounds identical to previous reports [14-26]: $m / z \quad 137 \rightarrow 111 \rightarrow 93$ for protocatechuic acid, $m / z \quad 337 \rightarrow 163 \rightarrow 145$ for neochlorogenic acid, $m / z \quad 121 \rightarrow 111 \rightarrow 93$ for protocatechualdehyde, $m / z \quad 121 \rightarrow 95 \rightarrow 77$ for $p$-hydroxybenzoic acid, $m / z \quad 337 \rightarrow 163 \rightarrow 145$ for chlorogenic acid, $m / z \quad 337 \rightarrow 163 \rightarrow 135$ for crypto-chlorogenin acid, $m / z \quad 163 \rightarrow 135 \rightarrow 123$ for caffeic acid, $m / z \quad 355 \rightarrow 311 \rightarrow 211 \rightarrow 179$ for swertiamarin, $m / z \quad 195 \rightarrow 179 \rightarrow 125$ for sweroside, $m / z \quad 545 \rightarrow 473 \rightarrow 443 \rightarrow 383 \rightarrow 353$ for schaftoside, $m / z$ $303 \rightarrow 285 \rightarrow 165 \rightarrow 137$ for agnuside, $m / z \quad 473 \rightarrow 443 \rightarrow 383 \rightarrow 353$ for isoschaftoside, $m / z \quad 473 \rightarrow 413 \rightarrow 293$ for flavosativaside, $m / z \quad 559 \rightarrow 547 \rightarrow 413 \rightarrow 293$ for vitexin $2^{\prime \prime}$-rhamnoside, $m / z \quad 300 \rightarrow 271 \rightarrow 179 \rightarrow 151$ for rutin, $m / z \quad 341 \rightarrow 323 \rightarrow 311 \rightarrow 283$ for vitexin, $m / z \quad 300 \rightarrow 271 \rightarrow 179 \rightarrow 151$ for hyperoside, $m / z$ $445 \rightarrow 401 \rightarrow 300 \rightarrow 271 \rightarrow 179$ for isoquercitrin, $m / z \quad 429 \rightarrow 383 \rightarrow 285$ for luteoloside, $\mathrm{m} / \mathrm{z}$ $499 \rightarrow 475 \rightarrow 429 \rightarrow 163$ for isochlorogenic acid $\mathrm{B}, \mathrm{m} / \mathrm{z} 535 \rightarrow 285 \rightarrow 255 \rightarrow 151$ for kaempferol-3-Orutinoside, $m / z \quad 499 \rightarrow 429 \rightarrow 163$ for isochlorogenic acid $\mathrm{A}, \mathrm{m} / \mathrm{z} \quad 417 \rightarrow 285 \rightarrow 239 \rightarrow 151$ for astragalin, $m / z \quad 267 \rightarrow 239 \rightarrow 151 \rightarrow 117$ for apigenin-7-glucoside, $m / z \quad 499 \rightarrow 429 \rightarrow 163$ for isochlorogenic acid C, $m / z \quad 237 \rightarrow 175 \rightarrow 151 \rightarrow 133$ for luteolin, $m / z \quad 273 \rightarrow 255 \rightarrow 151 \rightarrow 133$ for quercetin, $m / z 225 \rightarrow 151 \rightarrow 117$ for apigenin, and $m / z \quad 358 \rightarrow 343 \rightarrow 328 \rightarrow 300$ for casticin. Chlorogenic acid, rutin, schaftoside, and swertiamarin are used as examples to clarify the detailed identification processes of phenolic acids, $O$-glycosyl flavonoids, $C$-glycosyl flavonoids, and iridoid glycosides in Figure 2A-D, respectively. 
(A)

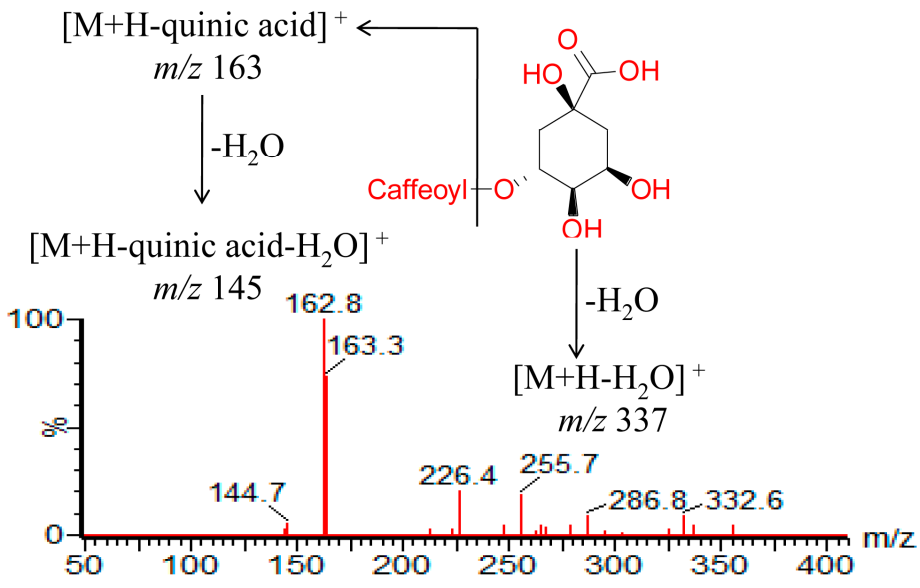

(B)

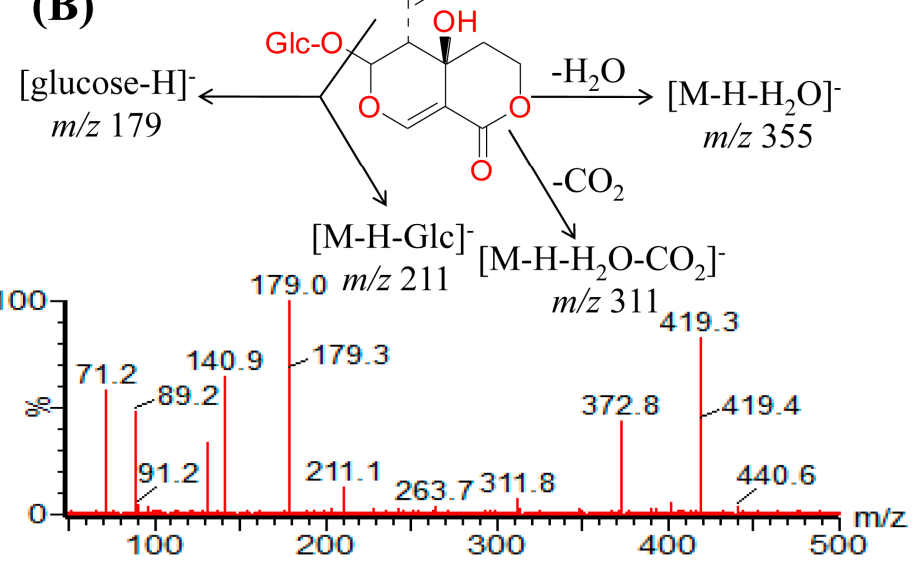

(C)

$0,2 \mathrm{X}_{0}-2 \mathrm{CH}_{2} \mathrm{O}\left[\mathrm{M}-\mathrm{H}-6 \mathrm{CH}_{2} \mathrm{O}\right]^{-}$
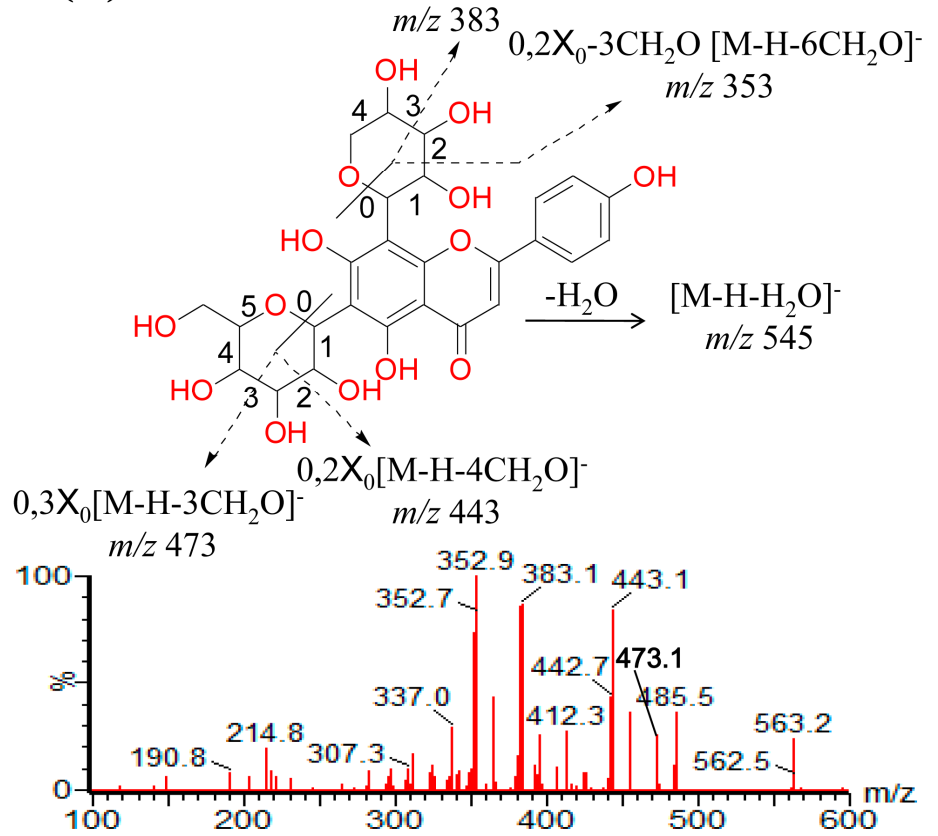

Figure 2. Cont. 


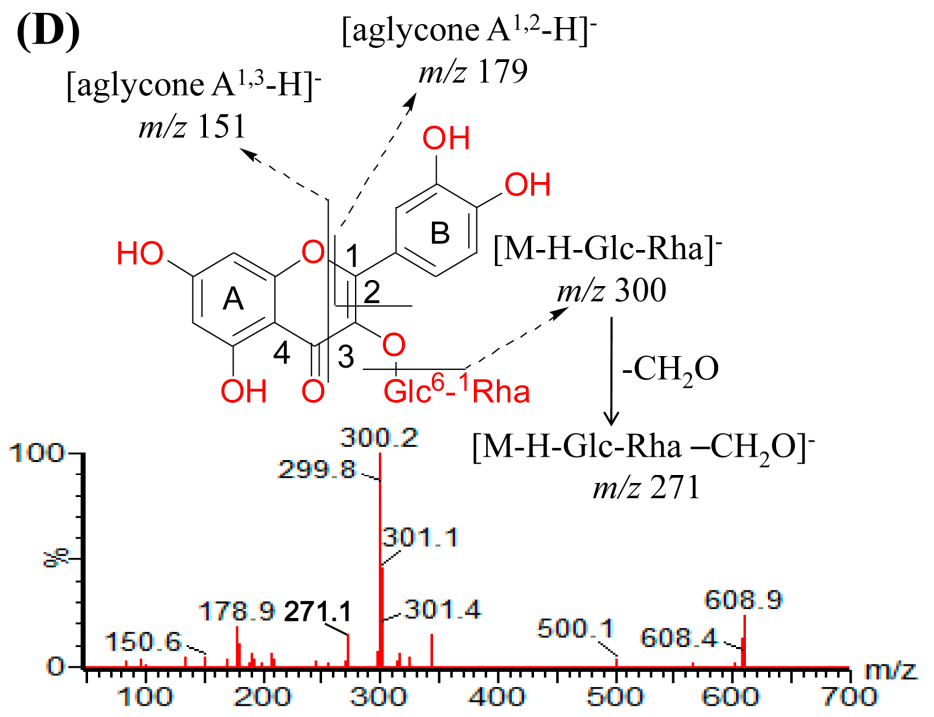

Figure 2. The ESI-MS/MS spectra and the proposed fragmentation pathway of chlorogenic acid (A); swertiamarin (B); schaftoside (C); and rutin (D).

\subsection{Method Validation}

(a) Linearity. The calibration curves, plotted with at least six concentrations of standard solutions, were constructed from the peak areas ratios of each standard to IS vs. concentration of each analyte. Acceptable linear correlation at these conditions was confirmed by correlation coefficients $(r, 0.9987-0.9998)$ (Figure 3).

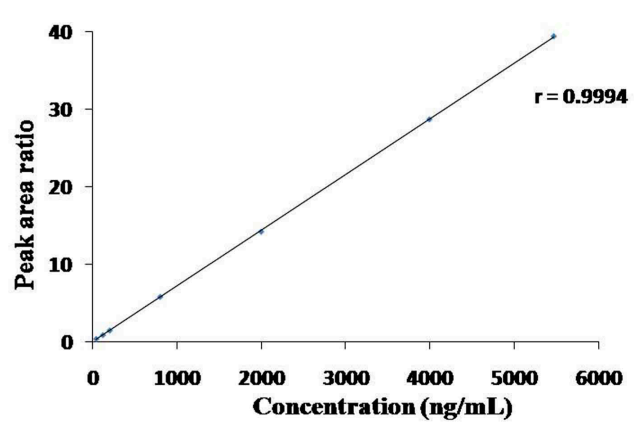

(A)

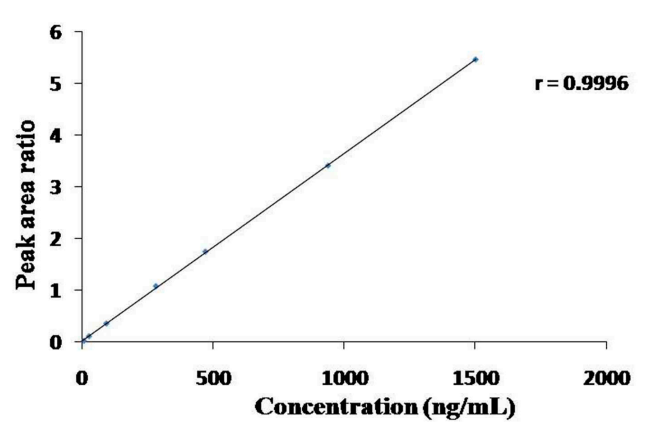

(B)

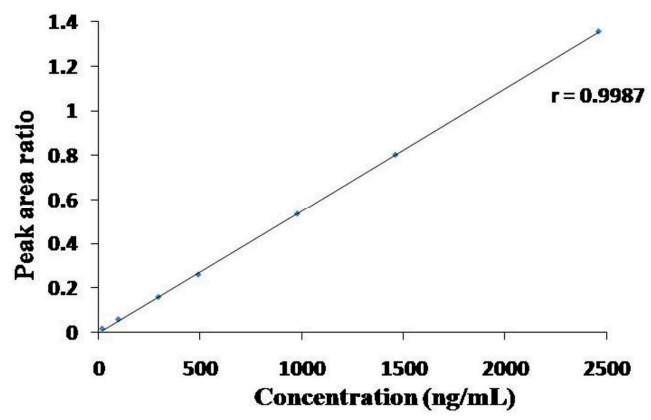

(C)

Figure 3. Linearity of representative compounds (chlorogenic acid (A); swertiamarin (B) and schaftoside $(\mathbf{C})$ ). 
(b) $L O D$ and $L O Q$. Limits of detection (LODs) and quantification (LOQs) are three times and ten times the noise level, respectively. For each target compound, the LODs and LOQs were determined by serial dilution of standard solution under the described UPLC-QqQ MS conditions. The LODs $(\mathrm{S} / \mathrm{N}=3)$ and LOQs $(\mathrm{S} / \mathrm{N}=10)$ for all standard analytes were in the range of $0.03-4.99$ and $0.16-14.87 \mathrm{ng} / \mathrm{mL}$, respectively, indicating that this method is sensitive for the quantitative determination of major components in YHKGT samples (Table S2, Supplementary Information).

(c) Precision. Intra- and inter-day variations were chosen to determine the precision from standard solutions the developed method. For intra-day precision test, the standards solutions were analyzed for six replicates within 1 day, while for inter-day precision test, the solutions were examined in duplicates for consecutive 3 days. The RSD values of intra- and inter-day precision were in the range of $0.84 \%-2.79 \%$ and $1.07 \%-4.87 \%$, respectively.

To confirm the precision from real samples (YHKGT), six samples of YHKGT (No. 1301014) were extracted and analyzed on three separate days. The RSD values of 29 standards were within the range of $2.03 \%-4.18 \%$. In order to investigate the stability of the samples, each sample solution was analyzed within $24 \mathrm{~h}(0,8,12$ and $24 \mathrm{~h})$ at room temperature. The RSD values of the 29 analytes were all less than $4.71 \%$ within $24 \mathrm{~h}$.

(d) Stability. Meanwhile, the stability of the standards was also investigated at $25^{\circ} \mathrm{C}$ standards were analyzed every $4 \mathrm{~h}$ within $12 \mathrm{~h}$ in triplicate. In conclusion, this developed method had good precision, repeatability and stability (Table 2 ).

Table 2. Precision, repeatability and stability of the 29 investigated compounds.

\begin{tabular}{|c|c|c|c|c|c|}
\hline \multirow[t]{2}{*}{ Compounds } & \multicolumn{2}{|c|}{$\begin{array}{l}\text { Precision from Standard } \\
\text { Solutions (RSD, } \%, n=6)\end{array}$} & \multirow{2}{*}{$\begin{array}{l}\text { Precision from Real } \\
\text { Samples (YHKGT) } \\
(\mathrm{RSD}, \%, n=6)\end{array}$} & \multirow[t]{2}{*}{ Stability (\%) } & \multirow{2}{*}{$\begin{array}{c}\text { Concentration } \\
(\mathbf{n g} / \mathbf{m L})\end{array}$} \\
\hline & Intra-day & Inter-day & & & \\
\hline Protocatechuic acid & 1.67 & 3.38 & 2.83 & $97.78 \pm 3.39$ & 399.52 \\
\hline Neochlorogenic acid & 1.29 & 3.21 & 2.27 & $102.45 \pm 3.03$ & 998.16 \\
\hline Protocatechualdehyde & 2.79 & 4.65 & 2.78 & $99.83 \pm 3.01$ & 39.95 \\
\hline$p$-Hydroxybenzoic acid & 1.56 & 2.63 & 2.03 & $98.9 \pm 2.95$ & 199.45 \\
\hline Chlorogenic acid & 1.70 & 3.55 & 2.34 & $98.34 \pm 2.14$ & 5992.3 \\
\hline Cryptochlorogenin acid & 2.01 & 3.98 & 2.76 & $103.12 \pm 3.84$ & 1996.54 \\
\hline Caffeic acid & 2.66 & 3.97 & 2.51 & $101.32 \pm 3.94$ & 39.95 \\
\hline Swertiamarin & 0.84 & 1.07 & 2.80 & $99.02 \pm 1.92$ & 19.97 \\
\hline Sweroside & 2.08 & 3.54 & 2.58 & $95.76 \pm 3.37$ & 998.6 \\
\hline Schaftoside & 1.34 & 1.99 & 3.63 & $98.37 \pm 2.68$ & 1996.22 \\
\hline Agnuside & 1.92 & 3.20 & 2.87 & $101.56 \pm 3.04$ & 599.36 \\
\hline Isoschaftoside & 1.12 & 2.54 & 2.67 & $98.45 \pm 2.43$ & 399.31 \\
\hline Flavosativaside & 1.01 & 1.63 & 2.08 & $102.89 \pm 3.29$ & 19.22 \\
\hline Vitexin $2^{\prime \prime}$-rhamnoside & 2.20 & 2.41 & 3.03 & $99.43 \pm 2.66$ & 19.67 \\
\hline Rutin & 0.87 & 1.51 & 2.33 & $102.36 \pm 2.02$ & 399.36 \\
\hline Vitexin & 1.94 & 2.75 & 2.54 & $99.43 \pm 2.39$ & 27.42 \\
\hline Hyperoside & 2.36 & 3.44 & 3.73 & $99.95 \pm 3.27$ & 50.13 \\
\hline Isoquercitrin & 1.23 & 2.42 & 4.18 & $95.72 \pm 2.56$ & 297.21 \\
\hline Luteoloside & 2.35 & 3.68 & 2.48 & $101.23 \pm 4.27$ & 195.87 \\
\hline Isochlorogenic acid B & 2.14 & 3.06 & 2.19 & $98.45 \pm 3.67$ & 998.82 \\
\hline
\end{tabular}


Table 2. Cont.

\begin{tabular}{|c|c|c|c|c|c|}
\hline \multirow[t]{2}{*}{ Compounds } & \multicolumn{2}{|c|}{$\begin{array}{l}\text { Precision from Standard } \\
\text { Solutions (RSD, } \%, n=6)\end{array}$} & \multirow{2}{*}{$\begin{array}{l}\text { Precision from Real } \\
\text { Samples (YHKGT) } \\
(\mathrm{RSD}, \%, n=6)\end{array}$} & \multirow[t]{2}{*}{ Stability (\%) } & \multirow{2}{*}{$\begin{array}{c}\text { Concentration } \\
(\mathrm{ng} / \mathrm{mL})\end{array}$} \\
\hline & Intra-day & Inter-day & & & \\
\hline Kaempferol-3-O-rutinoside & 1.94 & 3.37 & 4.10 & $99.59 \pm 3.64$ & 99.99 \\
\hline Isochlorogenic acid A & 2.56 & 4.87 & 2.99 & $96.31 \pm 4.54$ & 1997.97 \\
\hline Astragalin & 2.30 & 3.37 & 2.68 & $99.56 \pm 2.76$ & 99.1 \\
\hline Apigenin-7-glucoside & 1.86 & 2.89 & 3.16 & $103.45 \pm 3.08$ & 9.71 \\
\hline Isochlorogenic acid C & 1.22 & 3.90 & 2.93 & $96.34 \pm 3.45$ & 1996.38 \\
\hline Luteolin & 1.30 & 2.34 & 2.64 & $102.62 \pm 3.25$ & 28.25 \\
\hline Quercetin & 2.42 & 4.58 & 3.18 & $97.34 \pm 3.91$ & 196.3 \\
\hline Apigenin & 1.25 & 1.95 & 2.70 & $98.99 \pm 2.53$ & 9.61 \\
\hline Casticin & 2.04 & 3.76 & 2.99 & $101.25 \pm 3.29$ & 27.21 \\
\hline
\end{tabular}

(e) Accuracy. The recovery was used to evaluate the accuracy of the method and determine by adding the mixed standard solutions with three different concentration levels (low, medium and high) to the known amounts of YHKGT sample. Triplicate experiments were conducted at each level. The percentage recoveries were calculated according to the following equation: (detected amount - original amount) $\times 100 \%$ /spiked amount. As shown in Table 3, the recovery rate of 29 standards varied from 94.67 to $104.78 \%$ (RSDs $\leq 4.72 \%$ ), revealing the acceptable recovery and accuracy of this method.

Table 3. Recovery data of the proposed method $(n=3)$.

\begin{tabular}{|c|c|c|c|c|c|}
\hline Compounds & Original $(\mu \mathrm{g})$ & Spiked $(\mu \mathrm{g})$ & Detected $(\mu \mathrm{g})$ & Recovery (\%) & RSD (\%) \\
\hline \multirow{3}{*}{ Protocatechuic acid } & \multirow{3}{*}{17.36} & 31.2 & 49.97 & 104.52 & 3.59 \\
\hline & & 62.4 & 81.83 & 103.32 & 2.33 \\
\hline & & 156 & 180.13 & 104.34 & 2.78 \\
\hline \multirow{3}{*}{ Neochlorogenic acid } & \multirow{3}{*}{60.09} & 29.7 & 89.60 & 99.36 & 3.48 \\
\hline & & 59.4 & 122.32 & 104.76 & 3.13 \\
\hline & & 148.5 & 215.69 & 104.78 & 2.55 \\
\hline \multirow{3}{*}{ Protocatechualdehyde } & \multirow{3}{*}{4.27} & 29.9 & 35.07 & 103.01 & 3.97 \\
\hline & & 59.81 & 67.55 & 104.63 & 2.56 \\
\hline & & 149.52 & 150.14 & 97.56 & 3.08 \\
\hline \multirow{3}{*}{$p$-Hydroxybenzoic acid } & \multirow{3}{*}{12.47} & 27.6 & 40.14 & 100.25 & 3.86 \\
\hline & & 55.2 & 66.52 & 97.92 & 2.95 \\
\hline & & 138 & 155.98 & 103.99 & 2.90 \\
\hline \multirow{3}{*}{ Chlorogenic acid } & \multirow{3}{*}{365.29} & 29.85 & 395.29 & 100.50 & 3.40 \\
\hline & & 59.7 & 426.84 & 103.10 & 2.46 \\
\hline & & 149.24 & 514.22 & 99.79 & 2.14 \\
\hline \multirow{3}{*}{ Cryptochlorogenin acid } & \multirow{3}{*}{87.22} & 29.47 & 117.71 & 103.46 & 2.87 \\
\hline & & 58.94 & 148.96 & 104.75 & 3.73 \\
\hline & & 147.36 & 240.44 & 103.98 & 2.22 \\
\hline \multirow{3}{*}{ Caffeic acid } & \multirow{3}{*}{5.80} & 29.94 & 34.81 & 96.89 & 3.66 \\
\hline & & 59.89 & 63.03 & 96.89 & 2.87 \\
\hline & & 149.72 & 159.54 & 102.69 & 2.86 \\
\hline
\end{tabular}


Table 3. Cont.

\begin{tabular}{|c|c|c|c|c|c|}
\hline Compounds & Original $(\mu \mathrm{g})$ & Spiked $(\mu \mathrm{g})$ & Detected $(\mu \mathrm{g})$ & Recovery (\%) & RSD (\%) \\
\hline \multirow{3}{*}{ Swertiamarin } & \multirow{3}{*}{1.26} & 28.48 & 28.32 & 97.82 & 4.27 \\
\hline & & 56.96 & 57.10 & 98.03 & 2.00 \\
\hline & & 142.4 & 146.52 & 102.01 & 2.35 \\
\hline \multirow{3}{*}{ Sweroside } & \multirow{3}{*}{61.19} & 29.38 & 89.31 & 97.07 & 2.11 \\
\hline & & 58.75 & 120.47 & 100.90 & 2.34 \\
\hline & & 146.88 & 212.87 & 103.27 & 3.88 \\
\hline \multirow{3}{*}{ Schaftoside } & \multirow{3}{*}{85.0} & 29.7 & 116.03 & 101.11 & 2.40 \\
\hline & & 59.39 & 144.73 & 100.57 & 3.49 \\
\hline & & 148.48 & 232.67 & 99.45 & 2.84 \\
\hline \multirow{3}{*}{ Agnuside } & \multirow{3}{*}{30.10} & 29.94 & 58.91 & 96.23 & 3.12 \\
\hline & & 59.88 & 88.32 & 97.23 & 2.35 \\
\hline & & 149.7 & 186.40 & 103.74 & 2.33 \\
\hline \multirow{3}{*}{ Isoschaftoside } & \multirow{3}{*}{16.07} & 29.63 & 45.34 & 98.79 & 4.02 \\
\hline & & 59.26 & 72.80 & 97.42 & 2.47 \\
\hline & & 148.16 & 170.07 & 103.94 & 3.43 \\
\hline \multirow{3}{*}{ Flavosativaside } & \multirow{3}{*}{2.47} & 28.89 & 30.06 & 98.96 & 2.76 \\
\hline & & 57.78 & 58.70 & 97.32 & 2.50 \\
\hline & & 144.44 & 150.82 & 102.71 & 3.06 \\
\hline \multirow{3}{*}{ Vitexin 2"-rhamnoside } & \multirow{3}{*}{3.13} & 29.57 & 33.60 & 103.04 & 2.37 \\
\hline & & 59.14 & 62.91 & 101.08 & 3.10 \\
\hline & & 147.84 & 151.41 & 100.30 & 2.95 \\
\hline \multirow{3}{*}{ Rutin } & \multirow{3}{*}{17.87} & 30.07 & 48.32 & 101.26 & 2.21 \\
\hline & & 60.14 & 79.54 & 102.54 & 3.73 \\
\hline & & 150.36 & 173.59 & 103.56 & 4.07 \\
\hline \multirow{3}{*}{ Vitexin } & \multirow{3}{*}{1.07} & 41.22 & 43.04 & 101.82 & 3.44 \\
\hline & & 82.43 & 85.03 & 101.86 & 2.66 \\
\hline & & 206.08 & 215.73 & 104.16 & 3.96 \\
\hline \multirow{3}{*}{ Hyperoside } & \multirow{3}{*}{2.49} & 30.10 & 30.70 & 103.69 & 3.70 \\
\hline & & 60.19 & 60.32 & 101.06 & 2.60 \\
\hline & & 150.48 & 151.61 & 100.43 & 3.93 \\
\hline \multirow{3}{*}{ Isoquercitrin } & \multirow{3}{*}{12.12} & 29.81 & 42.54 & 102.05 & 4.72 \\
\hline & & 59.62 & 71.53 & 99.65 & 3.34 \\
\hline & & 149.05 & 162.91 & 101.17 & 3.63 \\
\hline \multirow{3}{*}{ Luteoloside } & \multirow{3}{*}{7.53} & 29.44 & 36.52 & 98.47 & 4.24 \\
\hline & & 58.88 & 68.42 & 103.41 & 4.59 \\
\hline & & 147.2 & 160.87 & 103.90 & 3.04 \\
\hline \multirow{3}{*}{ Isochlorogenic acid B } & & 27.72 & 93.59 & 102.34 & 4.37 \\
\hline & 65.22 & 55.44 & 119.99 & 98.79 & 2.84 \\
\hline & & 138.6 & 208.53 & 103.40 & 2.58 \\
\hline & & 30.07 & 48.32 & 101.26 & 3.21 \\
\hline Kaempferol-3-O-rutinoside & 17.87 & 60.14 & 79.54 & 102.54 & 2.73 \\
\hline & & 150.36 & 173.59 & 103.56 & 3.07 \\
\hline
\end{tabular}


Table 3. Cont.

\begin{tabular}{|c|c|c|c|c|c|}
\hline Compounds & Original $(\mu \mathrm{g})$ & Spiked $(\mu \mathrm{g})$ & Detected $(\mu \mathrm{g})$ & Recovery (\%) & RSD (\%) \\
\hline \multirow{3}{*}{ Isochlorogenic acid A } & \multirow{3}{*}{105.61} & 29.57 & 135.18 & 100.00 & 2.41 \\
\hline & & 59.14 & 165.23 & 100.81 & 3.07 \\
\hline & & 147.84 & 253.38 & 99.95 & 2.19 \\
\hline \multirow{3}{*}{ Astragalin } & \multirow{3}{*}{3.33} & 29.76 & 33.89 & 102.69 & 2.45 \\
\hline & & 59.52 & 65.60 & 103.88 & 2.86 \\
\hline & & 148.8 & 159.29 & 103.47 & 3.87 \\
\hline \multirow{3}{*}{ Apigenin-7-glucoside } & \multirow{3}{*}{0.56} & 29.16 & 30.63 & 103.12 & 3.98 \\
\hline & & 58.32 & 54.98 & 101.89 & 2.88 \\
\hline & & 145.8 & 131.72 & 98.88 & 2.24 \\
\hline \multirow{3}{*}{ Isochlorogenic acid $\mathrm{C}$} & \multirow{3}{*}{123.39} & 30.0 & 152.84 & 98.17 & 3.04 \\
\hline & & 60.0 & 185.91 & 102.53 & 2.36 \\
\hline & & 150.01 & 272.26 & 99.24 & 3.48 \\
\hline \multirow{3}{*}{ Luteolin } & \multirow{3}{*}{0.84} & 28.34 & 29.26 & 100.28 & 2.41 \\
\hline & & 56.68 & 55.10 & 96.97 & 2.32 \\
\hline & & 141.7 & 148.54 & 103.95 & 4.20 \\
\hline \multirow{3}{*}{ Quercetin } & \multirow{3}{*}{10.03} & 29.5 & 38.60 & 96.85 & 4.69 \\
\hline & & 59.01 & 67.48 & 97.36 & 2.55 \\
\hline & & 147.5 & 160.25 & 101.84 & 3.56 \\
\hline \multirow{3}{*}{ Apigenin } & \multirow{3}{*}{0.24} & 28.86 & 27.92 & 99.38 & 4.12 \\
\hline & & 57.72 & 56.93 & 98.22 & 3.15 \\
\hline & & 144.3 & 143.57 & 99.33 & 2.30 \\
\hline \multirow{3}{*}{ Casticin } & \multirow{3}{*}{2.94} & 27.3 & 29.03 & 97.03 & 3.40 \\
\hline & & 54.6 & 54.63 & 94.67 & 2.30 \\
\hline & & 136.5 & 142.14 & 101.98 & 2.65 \\
\hline
\end{tabular}

\subsection{Sample Analysis}

The validated method was successfully applied for the identification and quantification of 29 target compounds in 13 batches of YHKGT. The contents of the investigated compounds, based on their respective calibration curves, are summarized in Table 4. There were great variations among the contents of 24 compounds in different batches of YHKGT with RSD values exceeding 10\%. Among them, chlorogenic acid, isochlorogenic acid $\mathrm{A}$ and isochlorogenic acid $\mathrm{C}$, sweroside and agnuside, and schaftoside and rutin were the major phenolic acids, iridoids and flavonoids respectively, which have been found to have antiviral, anti-inflammatory and immunomodulatory properties [27-30]. Therefore, these three types of compounds should be considered as important bioactive components of YHKGT, and their content variabilities could influence the quality and efficacy of YHKGT. 
Table 4. Contents of 29 investigated compounds in 13 batches of YHKGT samples.

\begin{tabular}{lcccccccccccccccc}
\hline Samples & \multicolumn{1}{c}{ Content of Each Compound in 13 Batches of YHKGP Samples (mg/g) } \\
\hline No. & $\mathbf{1}$ & $\mathbf{2}$ & $\mathbf{3}$ & $\mathbf{4}$ & $\mathbf{5}$ & $\mathbf{6}$ & $\mathbf{7}$ & $\mathbf{8}$ & $\mathbf{9}$ & $\mathbf{1 0}$ & $\mathbf{1 1}$ & $\mathbf{1 2}$ & $\mathbf{1 3}$ & $\mathbf{1 4}$ & $\mathbf{1 5}$ \\
\hline 1208010 & 0.1580 & 0.5075 & 0.0186 & 0.1248 & 2.9787 & 0.7077 & 0.0211 & 0.0102 & 0.6596 & 0.9358 & 0.2806 & 0.1604 & 0.0056 & 0.0040 & 0.1847 \\
1209015 & 0.1636 & 0.4144 & 0.0214 & 0.0666 & 3.6644 & 0.5157 & 0.0287 & 0.0102 & 0.5304 & 0.4690 & 0.1894 & 0.0890 & 0.0050 & 0.0043 & 0.1984 \\
1211023 & 0.1467 & 0.3324 & 0.0236 & 0.0857 & 3.3666 & 0.5195 & 0.0270 & 0.0101 & 0.4447 & 0.6714 & 0.2512 & 0.1423 & 0.0051 & 0.0039 & 0.1920 \\
1301013 & 0.1652 & 0.5879 & 0.0268 & 0.0801 & 4.1785 & 0.7268 & 0.0480 & 0.0102 & 0.7163 & 0.5082 & 0.2279 & 0.1065 & 0.0056 & 0.0047 & 0.2528 \\
1301014 & 0.1192 & 0.2926 & 0.0130 & 0.0602 & 2.2661 & 0.4318 & 0.0151 & 0.0099 & 0.3370 & 0.6574 & 0.2410 & 0.1313 & 0.0064 & 0.0039 & 0.1998 \\
1302015 & 0.1367 & 0.5406 & 0.0196 & 0.0524 & 3.9758 & 0.7341 & 0.0317 & 0.0102 & 0.5921 & 0.5662 & 0.2158 & 0.0733 & 0.0050 & 0.0045 & 0.2423 \\
1302016 & 0.1636 & 0.5390 & 0.0202 & 0.0701 & 3.4165 & 0.6541 & 0.0231 & 0.0101 & 0.5479 & 0.5190 & 0.1880 & 0.0759 & 0.0054 & 0.0051 & 0.1972 \\
1302017 & 0.1685 & 0.6239 & 0.0274 & 0.0763 & 4.2379 & 0.7716 & 0.0371 & 0.0102 & 0.6172 & 0.5195 & 0.2091 & 0.1073 & 0.0050 & 0.0058 & 0.2065 \\
1302018 & 0.1698 & 0.5489 & 0.0189 & 0.0566 & 3.6544 & 0.7684 & 0.0273 & 0.0101 & 0.6357 & 0.4679 & 0.2182 & 0.0666 & 0.0049 & 0.0042 & 0.1871 \\
1304010 & 0.1693 & 0.5384 & 0.0304 & 0.0751 & 3.5976 & 0.6395 & 0.0235 & 0.0097 & 0.6612 & 0.5151 & 0.1797 & 0.0899 & 0.0048 & 0.0043 & 0.1979 \\
1304023 & 0.1396 & 0.5508 & 0.0174 & 0.0532 & 4.0312 & 0.6471 & 0.0292 & 0.0101 & 0.6498 & 0.4772 & 0.2347 & 0.0739 & 0.0050 & 0.0042 & 0.2095 \\
1304024 & 0.1717 & 0.5322 & 0.0175 & 0.1299 & 3.8052 & 0.7524 & 0.0267 & 0.0102 & 0.4648 & 0.8406 & 0.1597 & 0.1405 & 0.0049 & 0.0054 & 0.1987 \\
1304025 & 0.1509 & 0.4240 & 0.0153 & 0.0941 & 2.7936 & 0.5772 & 0.0163 & 0.0101 & 0.4603 & 0.5562 & 0.2440 & 0.0815 & 0.0052 & 0.0037 & 0.1745 \\
Aver. & 0.1556 & 0.4948 & 0.0208 & 0.0789 & 3.5359 & 0.6497 & 0.0273 & 0.0101 & 0.5629 & 0.5926 & 0.2184 & 0.1029 & 0.0052 & 0.0045 & 0.2032 \\
RSD (\%) & 9.89 & 19.22 & 23.23 & 30.35 & 15.63 & 16.19 & 30.31 & 1.34 & 18.80 & 23.86 & 14.62 & 29.13 & 8.07 & 13.59 & 10.73 \\
\hline
\end{tabular}


Table 4. Cont.

\begin{tabular}{lccccccccccccccc}
\hline Samples & \multicolumn{10}{c}{ Content of Each Compound in 13 Batches of YHKGP Samples (mg/g) } \\
\hline No. & $\mathbf{1 6}$ & $\mathbf{1 7}$ & $\mathbf{1 8}$ & $\mathbf{1 9}$ & $\mathbf{2 0}$ & $\mathbf{2 1}$ & $\mathbf{2 2}$ & $\mathbf{2 3}$ & $\mathbf{2 4}$ & $\mathbf{2 5}$ & $\mathbf{2 6}$ & $\mathbf{2 7}$ & $\mathbf{2 8}$ & $\mathbf{2 9}$ \\
\hline 1208010 & 0.0131 & 0.0248 & 0.1229 & 0.0767 & 0.5631 & 0.0477 & 0.9640 & 0.0420 & 0.0029 & 1.1698 & 0.0171 & 0.0898 & 0.0051 & 0.0156 \\
1209015 & 0.0074 & 0.0268 & 0.1084 & 0.0729 & 0.4345 & 0.0419 & 1.4652 & 0.0285 & 0.0034 & 1.1410 & 0.0121 & 0.0771 & 0.0051 & 0.0155 \\
1211023 & 0.0091 & 0.0257 & 0.1049 & 0.0585 & 0.4418 & 0.0654 & 1.3217 & 0.0494 & 0.0029 & 1.0473 & 0.0081 & 0.0728 & 0.0043 & 0.0152 \\
1301013 & 0.0078 & 0.0234 & 0.1156 & 0.0959 & 0.6249 & 0.0527 & 1.5898 & 0.0426 & 0.0024 & 1.6397 & 0.0143 & 0.0748 & 0.0046 & 0.0179 \\
1301014 & 0.0074 & 0.0261 & 0.1069 & 0.0623 & 0.4129 & 0.0636 & 1.2913 & 0.0502 & 0.0021 & 0.7809 & 0.0025 & 0.0701 & 0.0040 & 0.0152 \\
1302015 & 0.0054 & 0.0221 & 0.1015 & 0.0808 & 0.6398 & 0.0425 & 1.5587 & 0.0335 & 0.0027 & 1.1480 & 0.0044 & 0.0699 & 0.0040 & 0.0172 \\
1302016 & 0.0066 & 0.0214 & 0.1153 & 0.0879 & 0.4715 & 0.0391 & 1.4159 & 0.0325 & 0.0042 & 1.0685 & 0.0106 & 0.0706 & 0.0041 & 0.0163 \\
1302017 & 0.0078 & 0.0145 & 0.1033 & 0.0809 & 0.6676 & 0.0420 & 1.6356 & 0.0257 & 0.0029 & 1.2690 & 0.0075 & 0.0698 & 0.0041 & 0.0161 \\
1302018 & 0.0091 & 0.0209 & 0.1042 & 0.0741 & 0.5110 & 0.0378 & 1.4709 & 0.0269 & 0.0042 & 1.0873 & 0.0069 & 0.0676 & 0.0040 & 0.0162 \\
1304010 & 0.0064 & 0.0231 & 0.1144 & 0.0799 & 0.4860 & 0.0359 & 1.4387 & 0.0299 & 0.0039 & 1.1153 & 0.0064 & 0.0668 & 0.0040 & 0.0161 \\
1304023 & 0.0073 & 0.0233 & 0.1046 & 0.0874 & 0.6507 & 0.0410 & 1.5325 & 0.0273 & 0.0017 & 1.2526 & 0.0083 & 0.0723 & 0.0037 & 0.0174 \\
1304024 & 0.0087 & 0.0174 & 0.1080 & 0.0719 & 0.5950 & 0.0484 & 1.4747 & 0.0317 & 0.0031 & 0.9447 & 0.0109 & 0.0780 & 0.0035 & 0.0155 \\
1304025 & 0.0071 & 0.0189 & 0.0907 & 0.0614 & 0.4857 & 0.0473 & 0.9683 & 0.0361 & 0.0015 & 0.7741 & 0.0094 & 0.0748 & 0.0035 & 0.0167 \\
Aver. & 0.0080 & 0.0222 & 0.1077 & 0.0762 & 0.5373 & 0.0466 & 1.3944 & 0.0351 & 0.0029 & 1.1106 & 0.0091 & 0.0734 & 0.0042 & 0.0162 \\
RSD (\%) & 22.69 & 15.55 & 7.16 & 13.94 & 16.12 & 19.06 & 14.68 & 22.97 & 28.85 & 19.13 & 41.86 & 7.84 & 12.08 & 5.03 \\
\hline
\end{tabular}




\section{Experimental Section}

\subsection{Standards, Reagents and Materials}

Protocatechuic acid (1), neochlorogenic acid (2), protocatechualdehyde (3), 4-hydroxybenzoic acid (4), chlorogenic acid (5), cryptochlorogenin acid (6), caffeic acid (7), swertiamarin (8), sweroside (9), schaftoside (10), agnuside (11), isoschaftoside (12), flavosativaside (13), vitexin $2^{\prime \prime}$-rhamnoside (14), rutin (15), vitexin (16), hyperoside (17), isoquercitrin (18), luteoloside (19), isochlorogenic acid $\mathrm{B}$ (20), kaempferol-3-O-rutinoside (21), isochlorogenic acid A (22), astragalin (23), apigenin-7-glucoside (24), isochlorogenic acid C (25), luteolin (26), quercetin (27), apigenin (28), casticin (29), albiflorin (internal standard 1, IS1), liquiritin (internal standard 2, IS2) and 2-hydroxycinnamic acid (internal standard 3, IS3) were purchased from the Chinese National Institute for Control of Pharmaceutical and Biological Products (Beijing, China). The chemical structures of these compounds are shown in Figure 3. The purity of each reference standard was higher than $98 \%$ as determined by HPLC.<smiles>[R]C(=O)c1cc([R2])c(O)cc1[R]</smiles>

protocatechuic acid (1): $\mathrm{R}_{1}=\mathrm{H}, \mathrm{R}_{2}=\mathrm{OH}, \mathrm{R}_{3}=\mathrm{OH}$ protocatechualdehyde (3): $\mathrm{R}_{1}=\mathrm{H}, \mathrm{R}_{2}=\mathrm{OH}, \mathrm{R}_{3}=\mathrm{H}$ 4-hydroxybenzoic acid (4): $\mathrm{R}_{1}=\mathrm{H}, \mathrm{R}_{2}=\mathrm{H}, \mathrm{R}_{3}=\mathrm{OH}$<smiles>[R20]OC1C([R6])CC(O)(C([R20])=O)CC1[R20]</smiles>

neochlorogenic acid (2): $\mathrm{R}_{1}=\mathrm{H}, \mathrm{R}_{2}=\mathrm{H}, \mathrm{R}_{3}=$ caffeoyl, $\mathrm{R}_{4}=\mathrm{H}$ chlorogenic acid (5): $\mathrm{R}_{1}=$ caffeoyl, $\mathrm{R}_{2}=\mathrm{H}, \mathrm{R}_{3}=\mathrm{H}, \mathrm{R}_{4}=\mathrm{H}$ cryptochlorogenin acid (6): $\mathrm{R}_{1}=\mathrm{H}, \mathrm{R}_{2}=$ caffeoyl, $\mathrm{R}_{3}=\mathrm{H}, \mathrm{R}_{4}=\mathrm{H}$ isochlorogenic acid $B(20): R_{1}=$ caffeoyl, $R_{2}=$ caffeoyl, $R_{3}=H, R_{4}=H$ isochlorogenic acid $A$ (22): $R_{1}=$ caffeoyl, $R_{2}=H, R_{3}=$ caffeoyl, $R_{4}=H$ isochlorogenic acid $C(25): R_{1}=H, R_{2}=$ caffeoyl, $R_{3}=$ caffeoyl, $R_{4}=H$<smiles>CCOc1ccc(/C=C/C(=O)O)cc1O</smiles><smiles>CC(C)(C)[C@H]1C=C(COC(=O)c2ccc(O)cc2)C2C(OC3CCCCC3)C=COC21</smiles>

Figure 4. Cont. 
<smiles>[R]c1cc(-c2oc3c([R])c([R])c([R6])c([R5])c3c(=O)c2[R4])cc([R])c1[R]</smiles>

schaftoside (10): $\mathrm{R}_{1}=\mathrm{H}, \mathrm{R}_{2}=\mathrm{OH}, \mathrm{R}_{3}=\mathrm{R}_{4}=\mathrm{H}, \mathrm{R}_{5}=\mathrm{OH}, \mathrm{R}_{6}=C$-Glc, $\mathrm{R}_{7}=\mathrm{OH}, \mathrm{R}_{8}=C$-Ara isoschaftoside (12): $\mathrm{R}_{1}=\mathrm{H}, \mathrm{R}_{2}=\mathrm{OH}, \mathrm{R}_{3}=\mathrm{R}_{4}=\mathrm{H}, \mathrm{R}_{5}=\mathrm{OH}, \mathrm{R}_{6}=C$-Ara, $\mathrm{R}_{7}=\mathrm{OH}, \mathrm{R}_{8}=C$-Glc flavosativaside (13): $\mathrm{R}_{1}=\mathrm{H}, \mathrm{R}_{2}=\mathrm{OH}, \mathrm{R}_{3}=\mathrm{R}_{4}=\mathrm{H}, \mathrm{R}_{5}=\mathrm{OH}, \mathrm{R}_{6}=\mathrm{H}, \mathrm{R}_{7}=\mathrm{OH}, \mathrm{R}_{8}=\mathrm{C}^{-} \mathrm{Glc}^{4}$ - $^{1} \mathrm{Glc}$ vitexin-2-O-rhamnoside (14): $\mathrm{R}_{1}=\mathrm{H}, \mathrm{R}_{2}=\mathrm{OH}, \mathrm{R}_{3}=\mathrm{R}_{4}=\mathrm{H}, \mathrm{R}_{5}=\mathrm{OH}, \mathrm{R}_{6}=\mathrm{H}, \mathrm{R}_{7}=\mathrm{OH}, \mathrm{R}_{8}=C$-Glc ${ }^{2}{ }^{1} \mathrm{Rha}$ rutin (15): $\mathrm{R}_{1}=\mathrm{OH}, \mathrm{R}_{2}=\mathrm{OH}, \mathrm{R}_{3}=\mathrm{H}, \mathrm{R}_{4}=O-\mathrm{Glc}^{6}{ }_{-}{ }^{\mathrm{Rha}}, \mathrm{R}_{5}=\mathrm{OH}, \mathrm{R}_{6}=\mathrm{H}, \mathrm{R}_{7}=\mathrm{OH}, \mathrm{R}_{8}=\mathrm{H}$ $\operatorname{vitcxin}(16): \mathrm{R}_{1}=\mathrm{H}, \mathrm{R}_{2}=\mathrm{OH}, \mathrm{R}_{3}=\mathrm{R}_{4}=\mathrm{H}, \mathrm{R}_{5}=\mathrm{OH}, \mathrm{R}_{6}=\mathrm{H}, \mathrm{R}_{7}=\mathrm{OH}, \mathrm{R}_{8}=C$-Glc hyperoside (17): $\mathrm{R}_{1}=\mathrm{H}, \mathrm{R}_{2}=\mathrm{OH}, \mathrm{R}_{3}=\mathrm{H}, \mathrm{R}_{4}=\mathrm{O}-\mathrm{Gal}, \mathrm{R}_{5}=\mathrm{OH}, \mathrm{R}_{6}=\mathrm{H}, \mathrm{R}_{7}=\mathrm{OH}, \mathrm{R}_{8}=\mathrm{H}$ isoquercitrin (18): $\mathrm{R}_{1}=\mathrm{H}, \mathrm{R}_{2}=\mathrm{R}_{3}=\mathrm{OH}, \mathrm{R}_{4}=O-\mathrm{Glc}, \mathrm{R}_{5}=\mathrm{OH}, \mathrm{R}_{6}=\mathrm{H}, \mathrm{R}_{7}=\mathrm{OH}, \mathrm{R}_{8}=\mathrm{H}$ luteoloside (19): $\mathrm{R}_{1}=\mathrm{R}_{2}=\mathrm{OH}, \mathrm{R}_{3}=\mathrm{R}_{4}=\mathrm{H}, \mathrm{R}_{5}=\mathrm{OH}, \mathrm{R}_{6}=\mathrm{H}, \mathrm{R}_{7}=O$-Glc, $\mathrm{R}_{8}=\mathrm{H}$ kaempferol-3-O-rutinoside (21): $\mathrm{R}_{1}=\mathrm{H}, \mathrm{R}_{2}=\mathrm{OH}, \mathrm{R}_{3}=\mathrm{H}, \mathrm{R}_{4}=\mathrm{O}-\mathrm{Glc}^{6}-{ }_{-}{ }^{\mathrm{Rha}}, \mathrm{R}_{5}=\mathrm{OH}, \mathrm{R}_{6}=\mathrm{H}, \mathrm{R}_{7}=\mathrm{OH}, \mathrm{R}_{8}=\mathrm{H}$ astragalin (23): $\mathrm{R}_{1}=\mathrm{H}, \mathrm{R}_{2}=\mathrm{OH}, \mathrm{R}_{3}=\mathrm{H}, \mathrm{R}_{4}=O-\mathrm{Glc}, \mathrm{R}_{5}=\mathrm{OH}, \mathrm{R}_{6}=\mathrm{H}, \mathrm{R}_{7}=\mathrm{OH}, \mathrm{R}_{8}=\mathrm{H}$ apigenin-7-glucoside (24): $\mathrm{R}_{1}=\mathrm{H}, \mathrm{R}_{2}=\mathrm{OH}, \mathrm{R}_{3}=\mathrm{R}_{4}=\mathrm{H}, \mathrm{R}_{5}=\mathrm{OH}, \mathrm{R}_{6}=\mathrm{H}, \mathrm{R}_{7}=O$-Glc, $\mathrm{R}_{8}=\mathrm{H}$ luteolin (26): $\mathrm{R}_{1}=\mathrm{R}_{2}=\mathrm{OH}, \mathrm{R}_{3}=\mathrm{R}_{4}=\mathrm{H}, \mathrm{R}_{5}=\mathrm{OH}, \mathrm{R}_{6}=\mathrm{H}, \mathrm{R}_{7}=\mathrm{OH}, \mathrm{R}_{8}=\mathrm{H}$ quercetin (27): $\mathrm{R}_{1}=\mathrm{R}_{2}=\mathrm{OH}, \mathrm{R}_{3}=\mathrm{H}, \mathrm{R}_{4}=\mathrm{R}_{5}=\mathrm{OH}, \mathrm{R}_{6}=\mathrm{H}, \mathrm{R}_{7}=\mathrm{OH}, \mathrm{R}_{8}=\mathrm{H}$ apigenin (28): $\mathrm{R}_{1}=\mathrm{H}, \mathrm{R}_{2}=\mathrm{OH}, \mathrm{R}_{3}=\mathrm{R}_{4}=\mathrm{H}, \mathrm{R}_{5}=\mathrm{OH}, \mathrm{R}_{6}=\mathrm{H}, \mathrm{R}_{7}=\mathrm{OH}, \mathrm{R}_{8}=\mathrm{H}$ casticin (29): $\mathrm{R}_{1}=\mathrm{OH}, \mathrm{R}_{2}=\mathrm{OCH}_{3}, \mathrm{R}_{3}=\mathrm{H}, \mathrm{R}_{4}=\mathrm{OCH}_{3}, \mathrm{R}_{5}=\mathrm{OH}, \mathrm{R}_{6}=\mathrm{R}_{7}=\mathrm{OCH}_{3}, \mathrm{R}_{8}=\mathrm{H}$

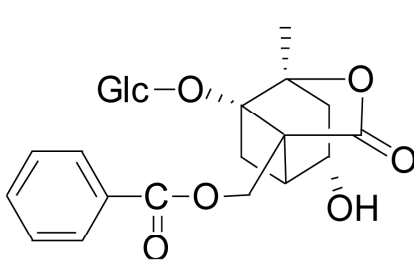

$\left(\mathrm{IS}_{1}\right)$ albiflorin<smiles>O=C(O)/C=C/c1ccccc1OC(Cl)Oc1ccc(C2CC(=O)c3ccc(O)cc3O2)cc1</smiles>

$\left(\mathrm{IS}_{2}\right)$ liquiritin

$\left(\mathrm{IS}_{3}\right)$ 2-hydroxycinnamic acid

Figure 4. Chemical structures of the 29 investigated compounds and three internal standards.

Acetonitrile, methanol and formic acid (HPLC grade) for UPLC analysis were bought from Merck (Darmstadt, Germany). Deionized water was prepared using a Millipore Milli-Q purification system (Millipore, Bedford, MA, USA). Thirteen batches of Yin Hua Kang Gan Tablet (YHKGT) samples were obtained from Zhangzhou PienTzeHuang Pharmaceutical Co. Ltd. (Zhangzhou City, Fujian Province, China) and stored at $4{ }^{\circ} \mathrm{C}$ until analysis. Voucher specimens were deposited in the College of Pharmacy, Fujian University of Traditional Chinese Medicine.

\subsection{Preparation of Standard Solution and Samples}

Stock solutions of the 29 standards (approx. $1 \mathrm{mg} / \mathrm{mL}$ ) were prepared individually by dissolving accurately weighted amount of standards in 70\% methanol-water. An internal standards stock solution was also prepared in a concentration of $0.32 \mu \mathrm{g} / \mathrm{mL}$ for liquiritin, $0.25 \mu \mathrm{g} / \mathrm{mL}$ for 2-hydroxycinnamic acid and $0.81 \mu \mathrm{g} / \mathrm{mL}$ for albiflorin. Then a mixed solution containing all the 29 standards were prepared and serially diluted with $70 \%$ methanol-water $(v / v)$ to obtain seven reference solutions with different concentrations used for plotting standard curves. All prepared solutions were stored at $4{ }^{\circ} \mathrm{C}$ 
before analysis. The 13 batches of YHKGT samples were ground to a fine powder. A powder sample $(0.20 \mathrm{~g})$ was accurately weighted and extracted with $100 \mathrm{~mL}$ of $70 \%$ methanol-water $(v / v)$ in an ultrasonic bath $(40 \mathrm{kHz}, 500 \mathrm{w})$ for $30 \mathrm{~min}$. Additional $70 \%$ methanol-water was added to make up the lost weight. The extracted solution was centrifuged at 150,000 rpm for $10 \mathrm{~min}$, and the supernatant was obtained as sample solution. The internal standard working solution $(500 \mu \mathrm{L})$ was added to $500 \mu \mathrm{L}$ of the mixed standards solution or sample solution, then vortex blended and filtered through a $0.22 \mu \mathrm{m}$ micropore membrane prior to injection. All the samples were stored at $4{ }^{\circ} \mathrm{C}$ before analysis.

\subsection{Liquid Chromatography}

Chromatographic analysis was performed on a Waters Acquity UPLC H-Class system (Milford, MA, USA) equipped with an online vacuum degasser, a binary pump, an autosampler, and a thermostated column compartment. Chromatographic separation was carried out at $45^{\circ} \mathrm{C}$ on an Waters Cortecs UPLC C18 column $(50 \times 2.1 \mathrm{~mm}, 1.6 \mu \mathrm{m})$. The mobile phases consisted of acetonitrile (A) and $5 \%$ methanol and $0.1 \%$ formic acid in water (B). The gradient elution program was as follows: $3 \%$ $\mathrm{A}$ at $0-2 \mathrm{~min}, 3 \%-7 \% \mathrm{~A}$ at $2-4 \mathrm{~min}, 7 \%-13 \% \mathrm{~A}$ at $4-6 \mathrm{~min}, 13 \%-20 \% \mathrm{~A}$ at $6-8 \mathrm{~min}, 20 \%-55 \% \mathrm{~A}$ at $8-8.5 \mathrm{~min}, 55 \%-90 \% \mathrm{~A}$ at $8.5-9.5 \mathrm{~min}, 90 \% \mathrm{~A}$ at $9.5-9.95 \mathrm{~min}, 90 \%-3 \% \mathrm{~A}$ at $9.95-10 \mathrm{~min}, 3 \% \mathrm{~A}$ at $10-12 \mathrm{~min}$. The flow rate was kept at $0.25 \mathrm{~mL} / \mathrm{min}$, and the injected sample volume was $2 \mu \mathrm{L}$.

\subsection{Mass Spectrometry}

Tandem mass spectrometry was performed on an Xevo TQ QqQ MS triple quadrupole mass spectrometer equipped with an electrospray ion source (ESI) (Waters). The MS spectra were acquired in multiple reaction monitoring (MRM) mode. Polarity switching electrospray ionization was applied. Argon was chosen as collision gas, nitrogen was chosen as nebulizer gas and heater gas. The MS conditions were optimized as follows: capillary voltage, $2.5 \mathrm{kV}$; source temperature, $200{ }^{\circ} \mathrm{C}$; dwell time, $20 \mathrm{~ms}$.

\section{Conclusions}

In this study, a UPLC-QqQ-MS method for the simultaneous determination of 29 major components in YHKGT has been developed and validated for the first time, which greatly improved its quality control. The polarity switching mode facilitated the detection of multiple types of constituents in YHKGT with different ionization responses. Compared with the current published HPLC method [13], this developed method enabled identification of target compounds with high selectivity by comparison with standards, high sensitivity and a rapid analysis was performed within $12 \mathrm{~min}$. The results obtained in this work demonstrated that polarity switching in UPLC-QqQ-MS provides an unsuspected advantage for complex method development in the TCM analytical services industry.

\section{Supplementary Materials}

Supplementary materials can be accessed at: http://www.mdpi.com/1420-3049/20/07/12209/s1. 


\section{Acknowledgments}

This study was supported by the National Natural Science Foundation of China (81373940); Provincial Natural Science Foundation of Fujian (2014J01352, 2012J05152, 2012Y4005); Provincial Health Department Foundation of Fujian (2013-2-55); Fujian University of Traditional Chinese Medicine Foundation (X2013015 and X2014132) and Fujian Key Laboratory of Chinese Materia Medica.

\section{Author Contributions}

$\mathrm{Yu}$ Lin and Wen $\mathrm{Xu}$ conceived and designed the experiments; Yu Lin, Mingqing Huang and Wei Xu performed the experiments; Huang Li and Miao Ye analyzed the data; Xun Zhang contributed reagents/materials/analysis tools; Yu Lin and Kedan Chu wrote the paper.

\section{Conflicts of Interest}

The authors declare that there are no conflicts of interest.

\section{References}

1. Cos, P.; Maes, L.; Vlietinck, A.; Pieters, L. Plant-derived leading compounds for chemotherapy of human immunodeficiency virus (HIV) infection-An update (1998-2007). Planta Med. 2008, 74, 1323-1337.

2. The State Pharmacopoeia Commission of the People's Republic of China. Drug Specifications for Traditional Chinese Medicine Promulgated by Ministry of Public Health of China; Chinese Pharmacopoeia Commission Press: Beijing, China, 1997; Volume 12, p. 169.

3. Qi, L.W.; Chen, C.Y.; Li, P. Structural characterization and identification of iridoid glycosides, saponins, phenolic acids and flavonoids in Flos Lonicerae Japonicae by a fast liquid chromatography method with diode-array detection and time-of-flight mass spectrometry. Rapid Commun. Mass Spectrom. 2009, 23, 3227-3242.

4. Park, S.H.; Roh, E.; Kim, H.S.; Baek, S.I.; Choi, N.S.; Kim, N.; Hwang, B.Y.; Han, S.B.; Kim, Y. Inhibition of IRAK-4 activity for rescuing endotoxin LPS-induced septic mortality in mice by lonicerae flos extract. Biochem. Biophys. Res. Commun. 2013, 442, 183-188.

5. Lan, W.; Jun, Z.Z.; Zesheng, Z. Characterization of antioxidant activity of extracts from Flos Lonicerae. Drug Dev. Ind. Pharm. 2007, 33, 841-847.

6. Liao, Y.; Dong, S.; Kiyama, R.; Cai, P.; Liu, L.; Shen, H. Flos lonicerae extracts and chlorogenic acid protect human umbilical vein endothelial cells from the toxic damage of perfluorooctane sulphonate. Inflammation 2013, 36, 767-779.

7. Ling, T.J.; Ling, W.W.; Chen, Y.J.; Wan, X.C.; Xia, T.; Du, X.F.; Zhang, Z.Z. Antiseptic activity and phenolic constituents of the aerial parts of Vitex negundo var. cannabifolia. Molecules 2010 , $15,8469-8477$.

8. Luo, Y.J.; Bian, Q.Q.; Zhang, Q.; Liu, Z.R.; Zhang, X.S. Separation of Chemical Compounds in Vitex negundo L. by Preparative High-speed Counter-current Chromatography. J. Instrum. Anal. 2011, 30, 1044-1049. 
9. Gautam, K.; Kumar, P.; Poonia, S. Larvicidal activity and GC-MS analysis of flavonoids of Vitex negundo and Andrographis paniculata against two vector mosquitoes Anopheles stephensi and Aedes aegypti. J. Vector Borne Dis. 2013, 50, 171-178.

10. Zhang, Y.; Yang, L.J.; Jiang, K.; Tan, C.H.; Tan, J.J.; Yang, P.M.; Zhu, D.Y. Glycosidic constituents from the roots and rhizomes of Melicope pteleifolia. Carbohydr. Res. 2012, 361, 114-119.

11. Li, Y.; Ooi, L.S.M.; Wang, H.; But, P.P.; Ooi, V.E.C. Antiviral activities of medicinal herbs traditionally used in southern mainland China. Phytother. Res. 2004, 18, 718-722.

12. Liao, Y.; Lin, A.; Xin, L.; Zhu, H.; Huang, C. Pharmacognostical studies on the stem and leaf of Mussaenda pubescens. Zhong Yao Cai 2000, 23, 195-197.

13. Guo, X.; Wu, Q.N.; Gong, Q.H. Determination of Chlorogenic acid in Yinhuakanggan Tablets by HPLC. Qilu Pharm. Affiairs 2005, 24, 283-284.

14. Han, Y.Q.; Wang, J.; Cui, Q.X.; Wang, L.Q.; Cheng, B.F.; Zhao, H.Z.; Jiang, M.; Bai, G.; Luo, G.A. Absorption, metabolism and effect of compatibility on absorption of qishenyiqi dropping pill. Biomed. Chromatogr. 2013, 28, 554-563.

15. Yang, Y.; Wang, H.J.; Yang, J.; Brantner, A.H.; Lower-Nedza, A.D.; Si, N.; Song, J.F.; Bai, B.; Zhao, H.Y.; Bian, B.L. Chemical profiling and quantification of Chinese medicinal formula Huang-Lian-Jie-Du decoction, a systematic quality control strategy using ultra high performance liquid chromatography combined with hybrid quadrupole-orbitrap and triple quadrupole mass spectrometers. J. Chromatogr. A 2013, 1321, 88-99.

16. An, H.; Wang, H.; Lan, Y.; Hashi, Y.; Chen, S. Simultaneous qualitative and quantitative analysis of phenolic acids and flavonoids for the quality control of Apocynum venetum L. leaves by HPLC-DAD-ESI-IT-TOF-MS and HPLC-DAD. J. Pharm. Biomed. Anal. 2013, 85, 295-304.

17. Wu, X.; Ding, W.; Zhong, J.; Wan, J.; Xie, Z. Simultaneous qualitative and quantitative determination of phenolic compounds in Aloe barbadensis Mill by liquid chromatography-mass spectrometry-ion trap-time-of-flight and high performance liquid chromatography-diode array detector. J. Pharm. Biomed. Anal. 2013, 80, 94-106.

18. Li, Q.M.; van den Heuvel, H.; Delorenzo, O.; Corthout, J.; Pieters, L.A.; Vlietinck, A.J.; Claeys, M. Mass spectral characterization of $\mathrm{C}$-glycosidic flavonoids isolated from a medicinal plant (Passiflora incarnata). J. Chromatogr. 1991, 562, 435-446.

19. Tomczyk, M.; Gudej, J.; Sochacki, M. Flavonoids from Ficaria verna Huds. Z. Naturforsch. C 2002, 57, 440-444.

20. Li, H.; Song, F.; Xing, J.; Tsao, R.; Liu, Z.; Liu, S. Screening and structural characterization of alpha-glucosidase inhibitors from hawthorn leaf flavonoids extract by ultrafiltration LC-DAD-MS(n) and SORI-CID FTICR MS. J. Am. Soc. Mass Spectrom. 2009, 20, 1496-1503.

21. Cuyckens, F.; Claeys, M. Mass spectrometry in the structural analysis of flavonoids. J. Mass Spectrom. 2004, 39, 1-15.

22. De Rijke, E.; Zappey, H.; Ariese, F.; Gooijer, C.; Brinkman, U.A. Liquid chromatography with atmospheric pressure chemical ionization and electrospray ionization mass spectrometry of flavonoids with triple-quadrupole and ion-trap instruments. J. Chromatogr. A 2003, 984, 45-58.

23. Granica, S.; Krupa, K.; Klebowska, A.; Kiss, A.K. Development and validation of HPLC-DAD-CAD-MS(3) method for qualitative and quantitative standardization of polyphenols in Agrimoniae eupatoriae herba (Ph. Eur). J. Pharm. Biomed. Anal. 2013, 86, 112-122. 
24. Hogner, C.; Sturm, S.; Seger, C.; Stuppner, H. Development and validation of a rapid ultra-high performance liquid chromatography diode array detector method for Vitex agnus-castus. J. Chromatogr. B Anal. Technol. Biomed. Life Sci. 2013, 927, 181-190.

25. Mathon, C.; Duret, M.; Kohler, M.; Edder, P.; Bieri, S.; Christen, P. Multi-targeted screening of botanicals in food supplements by liquid chromatography with tandem mass spectrometry. Food Chem. 2013, 138, 709-717.

26. Aberham, A.; Schwaiger, S.; Stuppner, H.; Ganzera, M. Quantitative analysis of iridoids, secoiridoids, xanthones and xanthone glycosides in Gentiana lutea L. roots by RP-HPLC and LC-MS. J. Pharm. Biomed. Anal. 2007, 45, 437-442.

27. Wang, G.F.; Shi, L.P.; Ren, Y.D.; Liu, Q.F.; Liu, H.F.; Zhang, R.J.; Li, Z.; Zhu, F.H.; He, P.L.; Tang, W.; et al. Anti-hepatitis B virus activity of chlorogenic acid, quinic acid and caffeic acid in vivo and in vitro. Antivir. Res. 2009, 83, 186-190.

28. Hao, B.J.; Wu, Y.H.; Wang, J.G.; Hu, S.Q.; Keil, D.J.; Hu, H.J.; Lou, J.D.; Zhao, Y. Hepatoprotective and antiviral properties of isochlorogenic acid A from Laggera alata against hepatitis B virus infection. J. Ethnopharmacol. 2012, 144, 190-194.

29. Suksamrarn, A.; Kumpun, S.; Kirtikara, K.; Yingyongnarongkul, B.; Suksamrarn, S. Iridoids with anti-inflammatory activity from Vitex peduncularis. Planta Med. 2002, 68, 72-73.

30. Savov, V.M.; Galabov, A.S.; Tantcheva, L.P.; Mileva, M.M.; Pavlova, E.L.; Stoeva, E.S.; Braykova, A.A. Effects of rutin and quercetin on monooxygenase activities in experimental influenza virus infection. Exp. Toxicol. Pathol. 2006, 58, 59-64.

Sample Availability: Samples of the compounds 1-29 are available from the authors.

(C) 2015 by the authors; licensee MDPI, Basel, Switzerland. This article is an open access article distributed under the terms and conditions of the Creative Commons Attribution license (http://creativecommons.org/licenses/by/4.0/). 